Physica 142B (1986) 253-262

North-Holland, Amsterdam

\title{
DYNAMICS OF PHASE SEPARATION: CLUSTER KINETICS AND SELF-SIMILARITY PROPERTY OF THE STRUCTURE FUNCTION*
}

\author{
J. MARRO ${ }^{\mathrm{a}}$ and R. TORAL ${ }^{\mathrm{a}, \mathrm{b}}$ \\ a Facultad de Física, Universidad de Barcelona, Diagonal 647, 08028-Barcelona, Spain \\ ${ }^{b}$ Physics Department, University of Edinburgh, JCMB, Mayfield Road, Edinburgh EH9 3JZ, UK
}

Received 1 July 1986

\begin{abstract}
We derive simple relations between the structure function, grain distribution and system excess energy during the phase separation processes occurring in a variety of mixtures suddenly cooled inside the miscibility gap. This allows to relate experimental observations performed by different techniques, namely small-angle scattering, microscopy and calorimetry, over a wide range of times and concentrations including the occurrence of percolation. We discuss in particular the self-similarity or dynamical scaling property of the structure function and other facts in the light of the proposed relations. In particular, we find no evidence of a behavior with time $R(t) \sim \operatorname{In}(t)$ for the mean grain size in alloys, except perhaps at very late times when it seems a small correction to the predominant behavior $R(t) \sim t^{1 / 3}$.
\end{abstract}

\section{Introduction}

An old, yet unresolved problem [1] of statistical mechanics in the exciting field of nonlinear phenomena far from equilibrium is the description of the process of phase separation which undergo many mixtures suddenly cooled from a homogeneous state to a temperature inside the miscibility gap [1-3]. The process, variously described as nucleation, spinodal decomposition, coarsening, Ostwald ripening and Smoluchowski coagulation, has also a great practical interest in metallurgy [4], surface science [5], and many other branches of physics and chemistry [6]. It can be observed in alloys, liquid and glassy mixtures, protein solutions, etc; cf. Refs. 1-17 for a bibliography. Those observations use scattering of $\mathrm{X}$-rays, light or neutrons in order to monitor the structure function $S(k, t)$ at time $t$ after quenching [9-17], electron or field ion microscopy to determine the properties of the grains, droplets or clusters in the precipitate [18-21], and calorimetry to evaluate the excess energy during the evolution [22]. The process of phase separation can also be simulated in a computer by using a lattice gas or binary alloy

\footnotetext{
* Partially supported by the U.S.-Spanish Cooperative Research program under grant CCB-8402/025.
}

model [23-26]. The computer experiments revealed in particular $[25,26]$ that fundamental quantities such as $S(k, t)$ remain self-similar during the system evolution towards equilibrium, a fact which has deserved recently a very detailed analysis, both theoretically $[27-33,8]$ and experimentally [9-21].

We investigate in this paper the relation between structure function, grain or cluster distribution and system excess energy during the relaxation of the system, thus extending a unifying idea already present in previous work, particularly in refs. $8,29,33$ and 34 . The relation worked out here should be useful to correlate experimental observations by different techniques, even in the occurrence of percolation, i.e. when one of the species concentration is large enough so that a (percolation) network extends throughout the whole system. We also discuss the self-similarity property in the light of that relation and conclude about the mechanisms driving the phase separation during the observable time regime.

The discussion can be made more precise by referring it to the lattice-gas version of the ferromagnetic Ising model [35] where the sites, $i=1 \ldots, N$, of a regular (say, simple cubic, $N=L^{3}$ ) lattice are either occupied by a particle $\left(n_{1}=1\right)$ or empty $\left(n_{1}=0\right)$, two particles at 
nearest-neighbor locations contributing an energy $-4 J, J>0$. The consideration of this model also allows here the use of good quality "data", which were obtained numerically elsewhere $[26,36]$, to test the validity of our results. It seems safe enough to suppose that our results should also hold in principle for more realistic systems including some ideal materials, as follows for instance from the detailed comparisons performed in refs. 8 and 33 . In any case they are expected to clarify the behavior of the kinetic lattice gas model, and we also compare our predictions with some experimental data corresponding to real materials.

\section{Cluster evolution and energy}

In the lattice-gas model, a cluster may be defined as a maximal set of connected particles, i.e. sites with $n_{1}=1$ related by nearest neighbor bonds. The size of a given cluster, $m$, is then defined as the number of particles belonging to it, and the energy, $S$, as the number of particlehole bonds (including both, surface and interior ones) incident on the cluster. We shall refer also to $S_{m}$, the average value of $S$ over all clusters of size $m$, and to $C_{m}$, the probability for the occurrence of a cluster of size $m$ in the system; $C(m, t)$ will denote $C_{m}$ at time $t$.

An interesting qualitative feature of $C(m, t)$ is that, after a short initial time following the quen$\mathrm{ch}$, the cluster distribution splits into two parts with no clusters of intermediate sizes present in the system. That is, one observes [36-39] a "gas phase" including monomers, dimers, etc., a "liquid phase" which consists of rather large clusters, say $m>m_{\mathrm{C}}$, and a well-defined gap at intermediate values of $m$; moreover, the gas phase remains quasi-stationary after that transient time $[38,39]$. The cluster distribution at equilibrium, i.e. the limit of $C(m, t)$ as $t \rightarrow \infty$, is described elsewhere $[40,41]$ for some representative values of $T$, the system temperature, and $\rho$,

$\rho=\sum_{m=1}^{\infty} m C(m, t)$ the system density which remains constant during the evolution. We shall attempt here, in particular, to relate the evolution with time of $C(m, t)$ to that of other relevant magnitudes in the system.

The behavior of $S_{m}$ with $m$ at equilibrium only depends on temperature; it can be worked out either exactly or very precisely for the threedimensional Ising model in the case of small values of $m$, say for $m \leqslant 20$ [41-43]. Those $S_{m}$ values are seen to follow approximately a simple power law, $S_{m}=S_{0} m^{\sigma}$, here one may neglect in practice the dependence of $\sigma$ on $T$; on this assumption, $\sigma$ is halfway [40] between the values $\sigma=2 / 3$, corresponding to compact spherical clusters, and $\sigma=1$, characterising a rather linear association. As $m$ increases to larger values, however, the surface becomes less dominant and one should expect $S_{m} \sim m^{2 / 3}, m \rightarrow \infty$ at low temperatures. That is, a convenient phenomenological description which is also very accurate, the accuracy increasing as $T$ is lowered, is

$S_{m}= \begin{cases}S_{0} m^{\sigma} & m \leqslant m_{\mathrm{c}} \\ S_{0} m_{\mathrm{c}}^{\sigma-2 / 3} m^{2 / 3} & m \geqslant m_{\mathrm{c}} .\end{cases}$

Table I lists some representative values for the parameters in this equation obtained numerically for the three-dimensional lattice-gas model. Note that the exact value for $m_{\mathrm{C}}$ and the fact that eq. (2.2) implies a too sharp transition from $\sigma>2 / 3$ to $\sigma=2 / 3$ are rather irrelevant here given that, as mentioned before, $C(m, t)$ is zero or negligible at intermediate values of $m$.

A very simple application of the above facts follows by considering the relation

Table I

Representative values of the parameters for eq. (2.2) in the case of the threedimensional lattice-gas model

\begin{tabular}{llll}
\hline$T / T$ & $S_{0}$ & $\sigma$ & $m_{\mathrm{c}}$ \\
\hline 0.59 & 5.50 & 0.844 & $\sim 50$ \\
0.78 & 5.60 & 0.860 & $\sim 90$ \\
0.89 & 6.02 & 0.870 & $\sim 150$ \\
\hline
\end{tabular}




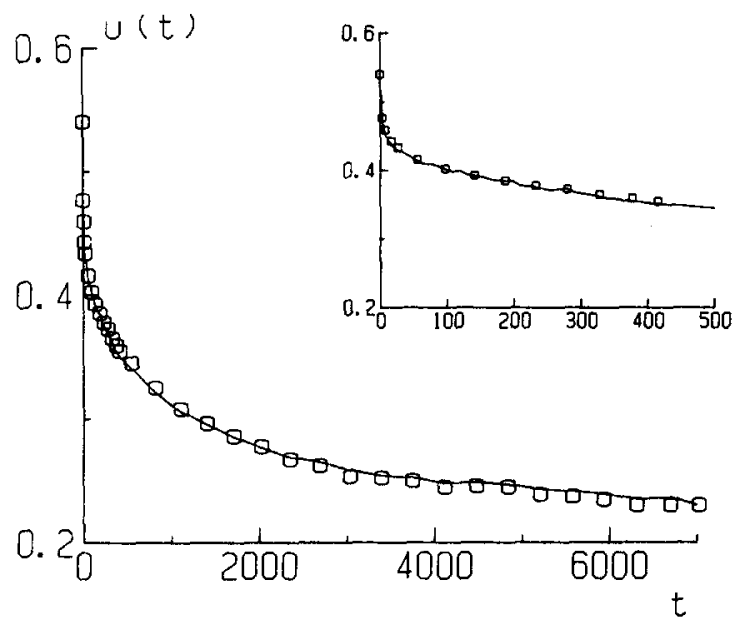

Fig. 1. Evolution with time, in Monte Carlo units, of the system energy measured as the number of particle-hole bonds per lattice site in the case of the three-dimensional lattice-gas model. The circles represent the direct result from a simulation at $\rho=0.10$ and $T / T_{\mathrm{G}}=0.6$; the lines follow from eqs. (2.3) and (2.2) with the parameters in table I. The inset shows the details at early times.

$u(t)=\sum_{m} C(m, t) S_{m}$,

where $u(t)$ represents the system energy measured as the number of (particle-hole) bonds per lattice site. Eq. (2.3) involves the assumption that the distribution of energies is practically time independent and equals the equilibrium distribution $S_{m}$. The use of numerical data for $C(m, t)$ and the expression (2.2) for $S_{m}$ in eq. (2.3) produce an excellent agreement with the temporal behavior of the energy in the case of the lattice-gas model for $T=0.6 T_{C}, \rho=0.01$ (see fig.1). The agreement is also very good for other phase points; sometimes one observes slight discrepancies for very initial times, but this seems just to reveal that $C(m, t)$ has no gap at intermediate values of $m$ for those times so that the sharp changeover contained in eq. $(2.2)$ is then a bad approximation to the actual, smooth situation.

In addition to eq. (2.3) it is also possible to write a useful relation between $u(t)$ and the structure function; this will be discussed in section 5 .

\section{Structure function and cluster properties}

The structure function may be defined here as the ensemble average of

$S(k, t)=N^{-1}\left|\sum_{j} \exp \left(\mathrm{i} \boldsymbol{k} \cdot \boldsymbol{r}_{j}\right) n\left(\boldsymbol{r}_{j}, t\right)\right|^{2}$,

where $N=L^{3}$ is the size of the system, $n\left(r_{i}\right) \equiv$ $n_{i}$ where $i$ runs over the $N$ sites of a simple cubic lattice, and the wave vector $k=(2 \pi / L) \mu, \mu_{\alpha}=$ $0, \pm 1, \cdots, \pm L / 2,(\alpha=1,2,3)$, is in the first Brillouin zone. This is essentially the Fourier transform of the correlation function:

$G(\boldsymbol{r}, t)=N^{-1} \sum_{\boldsymbol{r}^{\prime}} n\left(\boldsymbol{r}^{\prime}, t\right) n\left(\boldsymbol{r}^{\prime}+\boldsymbol{r}, t\right)$,

which may also be written as

$G(\boldsymbol{r}, t)=\sum_{m} m C(m, t) g(m, r)$.

Here $g(m, r)$ represents the (conditional) probability of having a particle at a distance $r$ apart from the origin (i.e. $n(r)=1$ ) when the origin $(r=0)$ belongs to an $m$-particle cluster. It then follows

$S(\boldsymbol{k}, t)=\sum_{m} m C(m, t) \hat{g}(m, \boldsymbol{k})$,

where

$\hat{g}(m, k)=\sum_{r} \mathrm{e}^{\mathrm{i} \boldsymbol{k} \cdot \boldsymbol{r}}[g(m, r)-\rho] ;$

$\rho$ is the system density defined as the mean values of $n_{i}$ in the system or, equivalently, as in eq. (2.1).

The function $g(m, r)$ (we are assuming an average over space directions consequently with the expected system isotropy) satisfies the properties:

$g(m, r=0)=1, \quad g(m, r=\infty)=\rho$

and, in the continuum limit,

$\int \mathrm{d} r[g(m, r)-\rho]=0:$ 
moreover, one also has

$g(m, 1)=1-\frac{S_{m}}{6 m}$,

where $S_{m} / 6 m$ is the mean number of particlehole bonds per particle and space direction in the case of $m$-particle clusters. The conditions (3.6)(3.8), however, cannot determine $g(m, r)$ completely. Fig. 2 depicts some simple assumptions on the behavior of $g(m, r)$ with $r$. The simplest one [34] assumes that $g(m, r)$ has a constant value for $r<r_{1}(m) \equiv(3 m / 4 \pi)^{1 / 3}$, corresponding to a spherical cluster of radius $r_{1}$, centered at the origin, and a different constant value, $\rho$, for $r>r_{2}(m) \equiv(3 m / 4 \pi \rho)^{1 / 3}$ while $g(m, r)=0$ for $r_{1}<r<r_{2}$. This "depletion zone" and the sharp transition at $r_{1}$ and $r_{2}$ are expected to represent rather unrealistically a mean behavior. Indeed, Binder et al. [34] showed how this assumption reproduces some interesting qualitative features of $S(k, t)$ but it failed to account for some other important facts; we shall come to this point later on. The actual $g(m, r)$ as defined in eq. (3.3) involves an average over cluster shapes implying a smooth behavior with $r$, e.g. the depletion zone should rather show up as a smooth

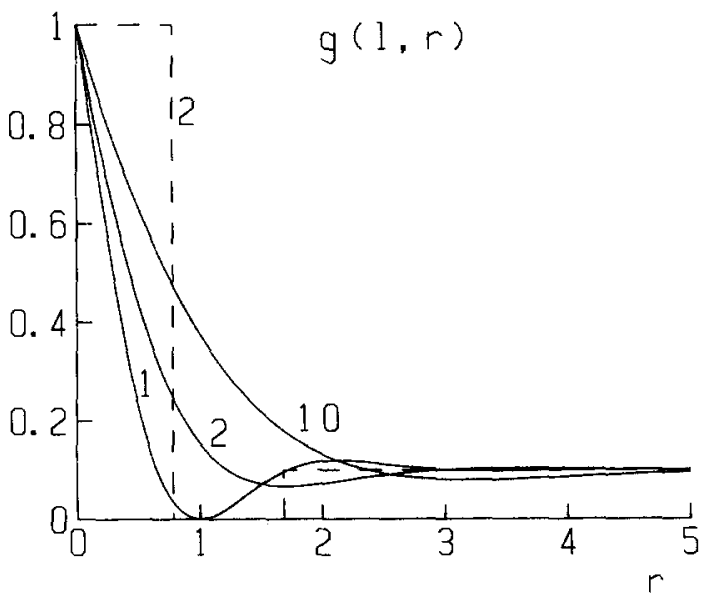

Fig. 2. The function $g(m, r)$, conditional probability of presence of a particle at a distance $r$ from the origin when the origin belongs to an $m$-particle cluster. The solid line is our assumption (3.9), with the parameters computed as explained in the main text, for different values of $m$, as indicated. The dashed line is the assumption in ref. 34 for $m=2$. minimun of $g(m, r)$ and this should have a continuous first derivate. A very simple assumption motivated by the above facts and by the work in ref. 34 is

$$
\begin{aligned}
g(m, r)= & \exp \left(-r / r_{1}\right)\left[A+(1-\rho-A) \cos \left(r / r_{2}\right)\right] \\
& +\rho,
\end{aligned}
$$

where $r_{1}, r_{2}$ and $A$ depend on $m$.

The parameters in eq. (3.9) can be determined from the above conditions. For instance, the sum rule (3.7) requires

$$
\begin{aligned}
& \frac{A}{A+\rho-1}=\frac{a^{4}\left(a^{2}-3\right)}{\left(1+a^{2}\right)^{3}}, \\
& a=a(m) \equiv \frac{r_{2}(m)}{r_{1}(m)} .
\end{aligned}
$$

On the other hand, $r_{2}(m)$ may be interpreted as measuring the mean radius of $m$-particle clusters (see fig. 2 and tables II), which is also related to $r_{1}(m)$. When $m \rightarrow \infty$, we may expand eq. (3.9) in powers of $\left(r_{1}\right)^{-1}$ and $\left(r_{2}\right)^{-1}$ for $r=1$ and, after using properties (3.8) and (2.2), it follows

$r_{1}(m)=\left[\frac{6(1-\rho)}{S_{0} m_{\mathrm{c}}^{\prime}}\right] m^{1 / 3}, \quad m \rightarrow \infty$

$m_{\mathrm{c}}^{\prime} \equiv\left(m_{\mathrm{c}}\right)^{\sigma-2 / 3}$. This suggests to consider

$r_{1}(m)=\left(a_{1}+b_{1} m\right)^{1 / 3}$

even for smaller values of $m$ than in eq. (3.11). The constant $b_{1}$ is determined by the limit condition $(3.11)$ as $b_{1}=\left[6(1-\rho) / S_{0} m_{c}^{\prime}\right]^{3}$, while $a_{1}$ follows from the fact that $g(1,1)=0$ which is in turn implied by eq. (3.8) and $S_{1}=6$ (coordination number of the simple cubic lattice), for instance. Moreover, one also knows that $g(m, r)$ is positively defined and that $\partial g(1, r) / \partial r$ equals zero at $r=1$ : these two conditions allow the computation of $r_{1}(1)$ and $r_{2}(1)$.

Now, one may attempt a consistent estimation of the parameters in eq. (3.9), leaving only one adjustable parameter free. To this end, we assume that the "mean radius" $r_{2}(m)$ behaves as 
Table II

Values of the parameter $r_{1}^{*} \equiv r_{1}\left(m^{*}\right)$ as a function of time as defined in eq. (3.17), and its comparison with $k_{1}(t)^{-1}$, the invers of the first moment (with a cut-off; see ref. 26) of the structure function, in the case of a Monte Carlo simulation at $T=0.6 T_{\mathrm{C}}$, $\rho=0.20$, i.e. in the presence of percolation. That proves that $r_{1}^{*}$ (and also $r_{2}^{*} \equiv r_{2}\left(m^{*}\right)$, since $r_{2}^{*}=\alpha^{1 / 3} r_{1}^{*}$ ) is proportional to $k_{1}(t)^{-1}$ as expected, see the text.

\begin{tabular}{lcccccccc}
\hline$t:$ & 108 & 572 & 1061 & 1588 & 2130 & 2972 & 3423 & 3897 \\
$r_{1}^{*}(t):$ & 1.577 & 2.158 & 2.431 & 2.586 & 2.742 & 2.941 & 3.068 & 3.198 \\
$k_{1}(t) r_{1}^{*}(t):$ & 1.43 & 1.50 & 1.51 & 1.51 & 1.51 & 1.52 & 1.52 & 1.52 \\
\hline
\end{tabular}

$r_{2}(m)=\left(a_{2}+b_{2} m\right)^{1 / 3}$

a condition which should indeed hold for large enough values of $m$. The constant $a_{2}$ can then be related immediately to $r_{2}(1)$ as estimated before, and $\alpha \equiv\left(b_{2} / b_{1}\right)^{1 / 3}$ can be chosen as the only free parameter in the description. Given that one also has $\alpha=\lim \left(r_{2} / r_{1}\right)$ as $m \rightarrow \infty$ and that $r_{1}(m)$ and $r_{2}(m)$ both have a geometrical meaning, we expect that $\alpha$ will be independent of density and temperature. Finally, the condition that $S(k, t)$ in eq. (3.4) should be positively defined, more specifically asking that $\partial S(k, t) / \partial k \geqslant 0$ at $k=0$, restricts the possible values of $\alpha$ to the range $1.165 \approx(5+\sqrt{52})^{1 / 2} / 3 \leqslant \alpha \leqslant \sqrt{3} \approx 1.73$.

The Fourier transform (3.5) can be written in the continuum limit as

$\hat{g}(m, k)=4 \pi k^{-1} \int_{0}^{\infty} \mathrm{d} r r \sin (k r)[g(m, r)-\rho]$

after performing the angular integrations. The only integral appearing here can also be performed when substituting eq. (3.9) for $g(m, r)$ giving

$$
\begin{gathered}
\hat{g}(m, k)=\frac{8 \pi A}{r_{1}\left(k^{2}+r_{1}^{-2}\right)^{2}}+4 \pi \frac{(1-\rho-A) r_{2}^{3}}{k r_{1}} \\
\quad \times\left[\frac{k r_{2}+1}{\left[\left(k r_{2}+1\right)^{2}+\alpha^{2}\right]^{2}}+\frac{k r_{2}-1}{\left[\left(k r_{2}-1\right)^{2}+\alpha^{2}\right]^{2}}\right] .
\end{gathered}
$$

On the other hand, using the expression by Binder et al. [34] for $g(m, r)$ in eq. (3.14) one would find instead $\hat{g}(m, k)=4 \pi k^{-3}\left[\tilde{a}\left(k, r_{1}\right)-\rho \tilde{a}\left(k, r_{2}\right)\right]$,

where

$\tilde{a}\left(k, r_{i}\right)=\sin \left(k r_{i}\right)-k r_{i} \cos \left(k r_{i}\right)$

and $r_{1}$ and $r_{2}$ differ from the ones in eq. (3.15). The expressions (3.15) and (3.16) for $\hat{g}(m, k)$ may be used in eq. (3.4) to obtain specific predictions for $S(k, t)$. In fig. 3 we compare the results following from those two assumptions with some Monte Carlo data for the lattice-gas model in the case $T=0.6 T_{\mathrm{C}}$ and $\rho=0.05$. While the assumption (3.16) leads to a systematic deviation from the data and produces negative values for $S(k, t)$ for large values of $k$, our expression (3.15) produces a good description;

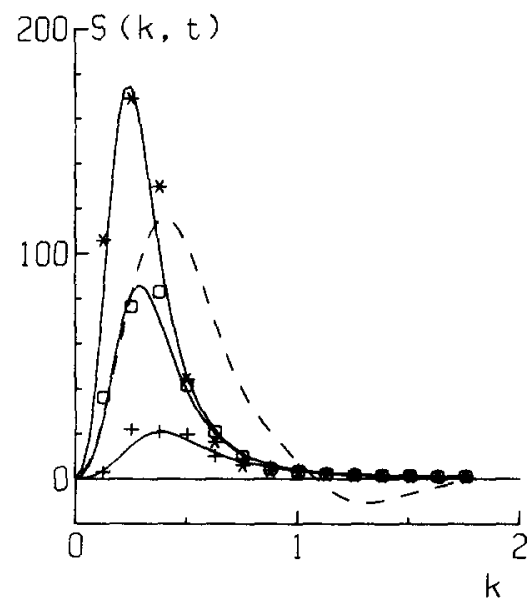

Fig. 3. Development of the structure function $S(k, t)$ with time. The solid lines correspond to our predictions in section 3 at times (from top to bottom): $t=13976, t=6012$ and $t=515$ in the case $T / T_{\mathrm{C}}=0.59$ and $\rho=0.05$. The symbols represent Monte Carlo data for the same values of the time, temperature and density. The dashed line corresponds to the assumption (3.16) for $t=6012$. 
indeed the small discrepancies one observes around the peak in this case may be interpreted as due to a fluctuation of the data which correspond to a single evolution and are thus severely affected by the finite size of the system [26, 8]. The situation for larger densities is similar, though the assumption (3.15) seems to provide better a description the lower the density is. This reflects the fact that eqs. (3.12) and (3.13) are too simple to describe increasing densities because the cluster shapes then become more and more difficult. That is, we believe that eq. (3.15) only needs to be completed with a more detailed estimation of $r_{1}(m)$ and $r_{2}(m)$ to provide an excellent prediction of $S(k, t)$, even for large values of $\rho$.

As a matter of fact, we shall show in the rest of this section, and in the next one, how one may obtain more pieces of useful information from the description above. To that end we shall attempt the computation of $S(k, t)$ on the assumption (3.9) in the case $T=0.6 T_{\mathrm{C}}, \rho=0.20$, where most of the particles in the system belong to a single, percolating cluster and eqs. (3.12)(3.13) are definitely inadequate. One may write in this case

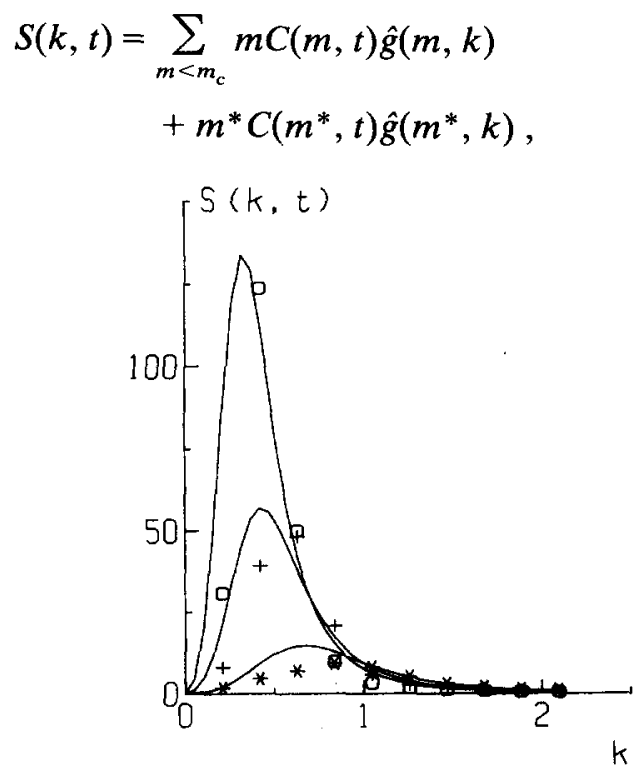

Fig. 4. Same as in fig. 3 for $T / T_{C}=0.59, \rho=0.2, t=3897$, 1061 and 108; after using eq. (3.17) as described in the main text. where $m^{*}$ represents the size of the percolating cluster, and consider $r_{1}\left(m^{*}\right)$ and $r_{2}\left(m^{*}\right)$ as two adjustable parameters while still requiring $r_{2}\left(m^{*}\right)=\alpha^{1 / 3} r_{1}\left(m^{*}\right)$; that is, $r_{2}\left(m^{*}\right)$ and $\alpha$ (in the range mentioned before) are now left free to adjust the Monte Carlo data. The resulting values for $r_{2}\left(m^{*}\right)$ are then proportional to $\left(k_{1}\right)^{-1}$, the inverse of the first moment of the structure function as reported in ref. 26 , thus confirming the consistency of the description (see table II). Fig. 4 depicts the temporal evolution of $S(k, t)$ as obtained from eq. (3.17).

\section{Self-similarity of the structure function}

The self-similarity or dynamical scaling property of the structure function reads $[25,26]$

$S(k, t)=k_{\mathrm{s}}(t)^{-3} F\left(k / k_{\mathrm{s}}(t)\right)$,

where $F(x)$ is some universal function and $k_{\mathrm{s}}(t)$ is a time-dependent scaling parameter related to some relevant physical length in the system such as the mean cluster radius, the location of the peak or the first moment of $S(k, t)$ etc. [8]. It is then possible to show that the assumption (3.9) is also consistent with the property eq. (4.1) which was previously demonstrated to hold for Monte Carlo data as well as for experimental data, and in the case of different interpretations for $\left(k_{\mathrm{s}}\right)^{-1}[8,26]$.

The simplest demonstration of this fact is by using the graphical method proposed by Fratzl et al. [8]. This consists in making plots of In $[S(k, t)]$ versus $\operatorname{In}(k)$ to determine two scaling parameters, one for each axis. Fig. 5 was prepared by applying this method to the function (3.4) and (3.15) with the parameters estimated as described in section 3 ; a similar result follows from eqs. (3.4) and (3.17) in the case of larger values of $\rho$. The figure clearly indicates that the property (4.1) is indeed contained in our formalism. Interesting enough, the resulting function $F(x)$ is consistent with Porod's law, namely $F^{-1} \sim x^{4}$, as shown by fig. 6 , and the scaling parameter for the largest density, $\rho=0.20$, is proportional to $r_{2}\left(m^{*}\right)$ which is in turn propor- 


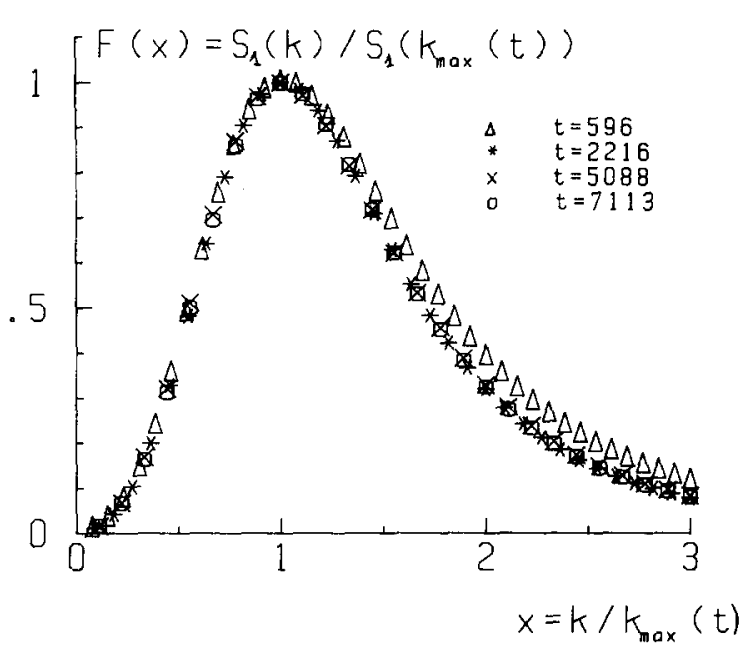

Fig. 5. The dynamical scaling function $F(x)$, as obtained from eqs. (3.4), (3.15) and (3.17) by following the graphical method described in ref. 8 in the case $T / T_{\mathrm{c}}=0.59, \rho=0.10$.

tional to $\left(k_{1}\right)^{-1}$ (see table II). It seems noticeable the fact that dynamical scaling holds the same at low densities (e.g. $\rho=0.05$ ) as in the presence of strong percolation effects (as for $\rho=0.20$ ) indicating that our approach suffers no fundamental limitations in that sense.

The fact that dynamical scaling is included in our formalism can be understood by noticing that one may write from eqs. (3.4) and (2.1),

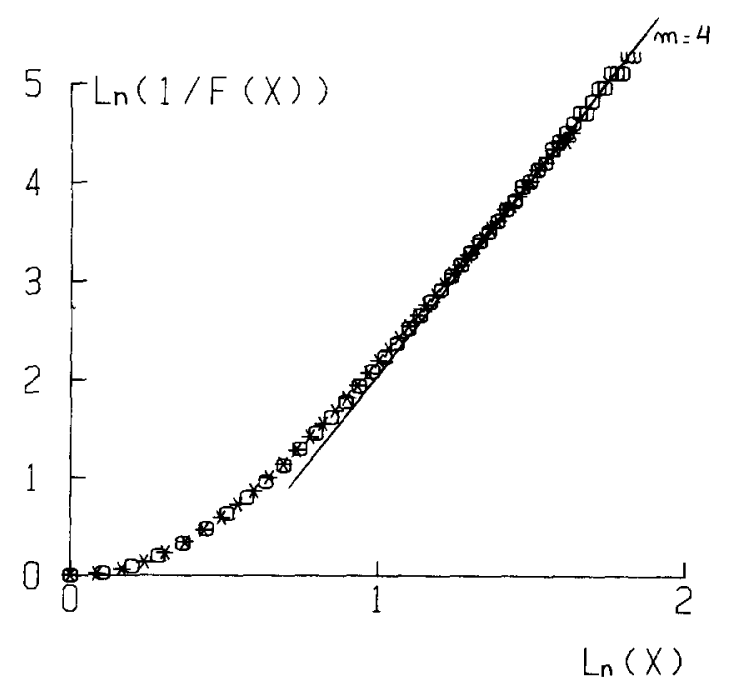

Fig. 6. The prediction for $F(x)$ in fig. 5 is plotted here to show the validity of Porod's law, $F^{-1} \sim x^{4}$.
$S(k, t)=\hat{g}(\bar{m}, k) \sum_{m} m C(m, t)=\rho \hat{g}(\bar{m}, k)$,

where $\bar{m}$ is some value of $m$ which, presumably, is near the maximum of the function $m \cdot C(m, t)$, i.e. near the mean cluster size. Thus, assuming $\hat{g}(\bar{m}, k)$ is given by eq. (3.15), we may write

$S(k, t)=r_{2}(\bar{m})^{3} F\left[k r_{2}(\bar{m})\right]$,

where

$$
\begin{aligned}
F(x)= & 4 \pi \rho A(\bar{m}) a(\bar{m})\left\{\frac{2}{\left(x^{2}+a(\bar{m})^{2}\right)^{2}}\right. \\
& +\frac{\left(1+a(\bar{m})^{2}\right)^{3}}{\left(3-a(\bar{m})^{2}\right) a(\bar{m})^{4}} \frac{1}{x} \\
& \times\left[\frac{x+1}{\left[(x+1)^{2}+a(\bar{m})^{2}\right]^{2}}\right. \\
& \left.\left.+\frac{x-1}{\left[(x-1)^{2}+a(\bar{m})^{2}\right]^{2}}\right]\right\}
\end{aligned}
$$

and $a(\vec{m})$ is defined as in eq. (3.10). The result (4.3)-(4.4) with a scaling parameter associated with the mean radius of the cluster maximizing the function $m C(m, t)$, agrees qualitatively with (4.1). The agreement of eq. (4.4), where there is no adjustable parameter, with data should only be expected qualitative in general given that, as discussed before, this description is too simple to incorporate every quantitative detail of phase separation. In any case, the above discussion reveals how a better knowledge of $C(m, t)$ in different systems could lead to an explicit evaluation of the universal function $F(x)$ within the present framework.

\section{System energy and structure function}

The energy, measured as the number of particle-hole bonds per lattice site, can be related to the structure function $[27,33]$, for instance, as

$$
\begin{aligned}
\Delta u(t)= & 2\left(\rho-\rho_{c}\right)\left(\rho+\rho_{\mathrm{c}}-1\right) \\
& \times\left[N^{-1} \sum_{k} j(k) S_{1}(k, t)-j(0)\right],
\end{aligned}
$$


where $\Delta u(t)=u(t)-u(\infty)$ is the excess energy, $S_{1}(k, t)$ represents the part of the structure function which is associated to the liquid phase [26] (this equals $S(k, t)$ in practice when the temperature is low enough, e.g. at $0.6 T_{C}$, excluding very early times), and $\rho_{\mathrm{C}}$ is the system density at the coexistence line, and $j(k)=\cos \left(k_{x}\right)+\cos \left(k_{y}\right)+$ $\cos \left(k_{z}\right), j(0)=3$, for nearest-neighbors interactions. After sphericalization one may simply write

$\sum_{k} j(k) S_{1}(k, t)=\frac{3 N}{16 \pi^{2}} \int_{0}^{2 \pi} \mathrm{d} k k \sin k S_{1}(k, t)$,

where the fact was used that $S_{1}(k, t)$ is typically negligible for $k \geqslant 2 \pi$ after a very short initial time. Moreover, the self-similarity property (4.1) may also be written as $S_{1}(k, t)=J_{G}(t) F(x), x=$ $k R_{G}(t), t>t_{S}$, where $R_{G}(t)$ is the scaling length obtained by following the Guinier method described in ref. 8. The substitution of this ansatz and eq. (5.2) in eq. (5.1) gives

$$
\begin{aligned}
\Delta u(t)= & 6\left(\rho-\rho_{\mathrm{C}}\right)\left(1-\rho-\rho_{\mathrm{C}}\right) \\
& \times\left[1-\frac{J_{\mathrm{G}}(t)}{R_{\mathrm{G}}^{2}(t)} \int_{0}^{2 \pi R_{\mathrm{G}}(t)} \mathrm{d} x x \sin \left(\frac{x}{R_{\mathrm{G}}}\right) F(x)\right]
\end{aligned}
$$

The facts that $F(x)$ decreases very sharply for $x>1$, that $R_{G}(t) \rightarrow$ const $\times L$ with $L$ the linear dimension of the system and $\Delta u(t) \rightarrow 0$ as $t \rightarrow \infty$, and that $J_{G}(t) / R_{G}(t)^{3} \propto N(\rho-\rho(t))$, where $\rho(t)$ represents the density of the gaseous phase at time $t$, allows one to simply write

$$
\Delta u(t)=6\left(1-\rho-\rho_{\mathrm{C}}\right)\left(\rho(t)-\rho_{\mathrm{C}}\right)
$$

which can be seen [33] to have the scaling form

$$
\Delta u(t)=\alpha f\left(\beta^{-1} t\right),
$$

where $\alpha$ and $\beta$ are density- and temperaturedependent parameters.

The results (5.4) and (5.5) were already seen to hold in the case of Monte Carlo [33] and microcalorimetric [22] experiments. Of course, a crucial point here is the specific time dependence in eqs. (5.4) and (5.5) [44]. A previous analysis [33] showed that all available experimental data, obtained either from microcalorimetric or numerical (Monte Carlo) experiments, strongly suggest that $\Delta u(t)^{-1}$ evolves at all times as a competition between two power laws, namely $t^{2 / 6}$ (as in the theory of Smoluchowski coagulation based on effective cluster diffusion [27]) and $t^{1 / 3}$ (as in the Ostwald ripening or LifshitzSlyozov monatomic diffusion [1]). That is, there is no time regime where the evolution is a consequence of either one or the other mechanism; instead one observes [33] two well-defined regimes: the initial one can be described as a combination of $t^{1 / 6}$ and $t^{1 / 3}$ - the former power law being relatively more important than the latter - and there is then a sharp transition to a second regime where the $t^{1 / 3}$ power law has a relative predominance. We performed a new analysis of the data for both real and model alloys looking for the behavior $\sim \operatorname{In}(t)$ at late times which is predicted by a renormalization group approach [31] and found that it seems rather irrelevant, for the behavior of both $\Delta u(t)^{-1}$ and $\left(k_{1}\right)^{-1}$, as compared to the behavior $t^{1 / 3}$. Thus, it seems that the conclusions in ref. 33 remain essentially unchanged for the observation times covered by the experiments (which are rather large when measured in the proper units [33]). The only evidence we find in favor of $R(t) \sim \operatorname{In}(t)$ is that the analysis of the data for very large times on the assumption that $\Delta u(t)^{-1} \sim \alpha t^{1 / 3}+\beta \cdot \operatorname{In}(t)$ seems to indicate that the last term represents a small correction to the predominant behavior $\sim t^{1 / 3}$ in the case of deep quenches. In any case, this correction is rather unimportant in practice as we also observe that the maximum of the structure function, $S\left(k_{\max }(t), t\right)$, follows very accurately a linear behavior with $t$.

\section{Conclusions}

The evolution with time of the structure function $S(k, t)$, cluster distribution $C(m, t)$ and system energy $u(t)$ reveals some of the most impor- 
tant features of phase separation in a quenched mixture. We investigate in this paper the relation between these magnitudes, thus connecting their properties in a way which should in principle be useful in the analysis of the experimental data obtained by different techniques, such as smallangle scattering, microscopy and calorimetry, and for the development of theory.

More specifically, we showed explicity (fig. 1) that it follows the behavior of $u(t)$ in agreement with Monte Carlo data by combining the known data for $C(m, t)$ via eq. (2.3). The behavior of $S(k, t)$ can also be obtained from that for $C(m, t)$ by introducing the function $g(m, r)$ as defined in eq. (3.3).

With that aim we introduced the assumption (3.9) (fig. 2) for $g(m, r)$ and computed consistently the corresponding parameters for small values of the system density $\rho$ under the hypothesis (3.12)-(3.13); this leaves just one adjustable parameter, $\alpha \equiv\left(b_{2} / b_{1}\right)^{1 / 3}$, which can only vary within a narrow range, $1.17 \leqslant \alpha \leqslant 1.73$. Eq. (3.15) then follows producing very good estimates for $S(k, t)$ at low $\rho$ (fig. 3 ). When $\rho$ is large enough, the system undergoes percolation; eq. (3.9) remains valid while one needs to remove (3.12)-(3.13) in this case. Yet, one may obtain $S(k, t)$ in reasonable agreement with the Monte Carlo data (fig. 4) by treating separately the large, "infinite" cluster of mean size $m^{*}$ as in eq. (3.17); one has $r_{2}\left(m^{*}\right)=\alpha{ }^{1 / 3} r_{1}\left(m^{*}\right)$ where $r_{2}\left(m^{*}\right)$ and $\alpha$ (the latter within the range mentioned above) are adjustable parameters. The resulting value of $r_{2}\left(m^{*}\right)$ as a function of time is proportional (table II) to $k_{1}(t)^{-1}$, the inverse of the first moment of $S(k, t)$ as obtained from Monte Carlo data, revealing it has an actual physical relevance as a measure of the mean cluster radius.

We also show that the self-similarity or dynamical scaling property of $S(k, t)$ is contained (figs. 5 and 6) in our formalism, i.e. it is consistent with the simple assumption (3.9), and give and explicit expression for the corresponding scaling function $F(x)$, eq. (4.4); this contains Porod's law $F(x)^{-1} \sim x^{4}$ for large values of $x$.

For completeness, we rederive some fun- damental scaling properties, eqs. (5.4) and (5.5), for the evolution with time of the excess energy $u(t)$ which are implied by the properties of $S(k, t), F(x)$ and $C(m, t)$ [33]. This allows to relate experimental (microcalometric) and Monte Carlo data to each other, i.e. they all lie on a single curve when one scales appropriately the corresponding units for $u$ and $t[22,33]$. By performing such a global analysis of most available data for $\Delta u$ corresponding to real and model alloys, it follows that the system relaxation may be described quite consistently assuming there are two well-defined regimes, both involving a competition between $t^{1 / 6}$ (effective cluster diffusion) and $t^{1 / 3}$ (monatomic diffusion) power laws. It is observed during the initial regime that $t^{1 / 6}$ dominates while $t^{1 / 3}$ is an important correction; during the final regime, on the contrary, the evolution proceeds according to $t^{1 / 3}$ slightly modified by $t^{1 / 6}$. The separation between those two regimes is rather sharp, the transition time depends on $\rho$ and $T$, and it occurs sooner the deeper the quench is. We performed a similar analysis looking for some evidence of a behavior $\sim \operatorname{In}(t)$, as predicted in ref. 31 ; we only found a weak evidence that it might be a small correction to the behavior $t^{1 / 3}$ in the case of deep quenches at very late values of the time.

Finally, we also notice that a part of our analysis in this paper heavily rests upon the knowledge of $C(m, t)$. This results in the fact that the present description of phase separation can easily incorporate kinetic equations for $C(m, t)$. For instance, the equations developed in refs. 38 and 39 for the time evolution of $C(m, t)$ may be combined with eqs. (3.4) and (3.15) to obtain the evolution of $S(k, t)$ as the system relaxes towards equilibrium. The numerical treatment of those equations, which provides a very accurate description of $C(m, t)$ at low densities, requires however a large amount of computer time [39]. As a consequence, in order to obtain in practice the behavior of $S(k, t)$ from the kinetic equations for the cluster distribution, one needs simpler kinetic equations and more economical methods of solution than before; this problem is investigated elsewhere [45]. 


\section{References}

[1] For early references, see R. Becker and W. Doring, Ann. Phys. (Leipzig) 24 (1935) 719. I.M. Lifshitz and V.V. Slyozov, J. Phys. Chem. Solids 19 (1961) 35. J.W. Cahn, Trans. AIME 242 (1968) 166. J.S. Langer, M. Baron and H.D. Miller, Phys. Rev. A11 (1975) 1417. J.D. Gunton, M. San Miguel and P.S. Sahni, in: Phase Separation and Critical Phenomena, C. Domb and J.L. Lebowitz, eds. (Academic Press, London, 1983).

[2] J.L. Lebowitz, J. Marro and M.H. Kalos, Comments on Sol. State Phys. 10 (1983) 201-17.

[3] J.D. Gunton and M. Droz, Introduction to the Theory of Metastable and Unstable States (Lecure Notes in Physics, Vol. 183), (Springer-Verlag, Berlin, 1983).

[4] B. Ditchek and L.H. Schwartz, Ann Rev. Mat. Sci. 9 (1979) 219-53.

[5] G.C. Wang and T.M. Lu, Phys. Rev. Lett. 50 (1983) 2014.

[6] G.C. Zettlemoyer, ed., Nucleation III (Advances in Colloid and Interface Science, Vol. 10), (Elsevier, Amsterdam, 1983).

[7] W.I. Golburg, in: Scattering Techniques Applied to Supramolecular and Nonequilibrium Systems, S. Chen, B. Chu and R. Nossal, eds. (Plenum Press, New York, 1981) pp. 383-409.

[8] P. Fratzl, J.L. Lebowitz, J. Marro and M.H. Kalos, Acta Metall. 31 (1983) 1849.

[9] S. Katano and M. Lizumi, Phys. Rev. Lett. 52 (1984) 835 .

[10] J.P. Simon, P. Guyot and A. Ghilarducci de Salva, Philos. Mag. A49 (1984) 151.

[11] S. Komura, K. Osamura, H. Fujii and T. Takeda, Phys. Rev. B30 (1984) 2944-7.

[12] O. Blaschko and P. Fratzl, Phys. Rev. Lett. 51 (1983) 288-91.

[13] A. Craievich and J.M. Sanchez, Phys. Rev. Lett. 47 (1981) 1308.

[14] Y. Yamada, N. Hamaya, J.D. Axe and S.M. Shapiro, Phys. Rev. Lett. 53 (1984) 1665-8.

[15] Y.C. Chou and W.I. Goldburg, Phys. Rev. A23 (1981) 858-64.

[16] J.K. Hoffer, L.J. Campbell and R.J. Bartlett, Phys. Rev. Lett. 45 (1980) 912-5.

[17] C.M. Knobler and N.C. Wong, J. Chem. Phys. 85 (1981) 1972.

[18] Y. Seno, Y. Tomokiyo, K. Oki and T. Eguchi, Trans. Japan Inst. Metals 24 (1983) 491-8.

[19] S. Krishnamurthy and W.I. Goldburg, Phys. Rev. A22 (1981) 2147-55.
[20] R.J. Rioja and D.E. Laughlin, Acta Met. 28 (1980) 1301.

[21] T. Abe, K. Miyazaki and K.I. Hirano, Acta Metall. 30 (1982) 357.

[22] J. Marro, R. Toral and A.M. Zahra, J. Phys. C18 (1985) 1377-86.

[23] G.S. Grest and D.J. Srolovitz, Phys. Rev. B30 (1984) 5150-5.

[24] A. Sadiq and K. Binder, J. Stat. Phys. 35 (1984) 517-85.

[25] J. Marro, J.L. Lebowitz and M.H. Kalos, Phys. Rev. Lett. 23 (1979) 282.

[26] J.L. Lebowitz, J. Marro and M.H. Kalos, Acta Metall. 30 (1982) 297.

[27] K. Binder and D. Stanffer, Phys. Rev. Lett. 33 (1974) 1006. K. Binder, Phys. Rev. B15 (1977) 4425.

[28] H. Furukawa, Phys. Rev. Lett. 43 (1979) 136; Phys. Rev. A23 (1981) 1535; Phys. Rev. A28 (1983) 1717-28.

[29] P.A. Rikvold and J.D. Gunton, Phys. Rev. Lett. 49 (1982) 286.

[30] T. Ohta, D. Jasnow and K. Kawasaki, Phys. Rev. Lett. 49 (1982) 1223.

[31] G.F. Mazenko and O.T. Valls, Phys. Rev. Lett. 51 (1983) 2044. G.F. Mazenko, O.T. Valls and F.C. Zhang, Phys. Rev. B32 (1985) 5807-17.

[32] M. Kolb, Phys. Rev. Lett. 53 (1984) 1653-6.

[33] R. Toral and J. Marro, Phys. Rev. Lett. 54 (1985) 1424-7.

[34] K. Binder, C. Billotet and P. Mirold, Z. Phys. B30 (1978) 183.

[35] C.J. Thompson, Mathematical Statistical Mechanics (Princeton Univ. Press, Princeton, NJ, 1972).

[36] K. Binder, M. Kalos, J. Lebowitz and J. Marro, Adv. Coll. Interf. Sci. 10 (1979) 173-214.

[37] A. Sur, J. Lebowitz, J. Marro and M. Kalos, Phys. Rev. B15 (1977) 3014-26.

[38] O. Penrose, J. Lebowitz, J. Marro and M. Kalos, J. Stat. Phys. 19 (1978) 243-67.

[39] O. Penrose, J. Lebowitz, J. Marro, M. Kalos and J. Tobochnik, J. Stat. Phys. 34 (1984) 399-426.

[40] J. Marro and R. Toral, Surf. Sci. 172 (1986) L539.

[41] J. Marro and R. Toral, Physica 122A (1983) 563-586.

[42] G. Jacucci, A. Perini and G. Martin, J. Phys. A16 (1983) 369.

[43] A. Perini, G. Jacucci and G. Martin, Phys. Rev. B29 (1984) 2689.

[44] J.D. Gunton, Communic. to the 9th Sitges Conf. on Fluctuations and Stochastic Phenomena in Condensed Matter, Sitges, Barcelona (May 1986), Proc. to be pub. lished by Springer-Verlag, Berlin.

[45] R. Toral and J. Marro, submitted to J. Phys. C. 